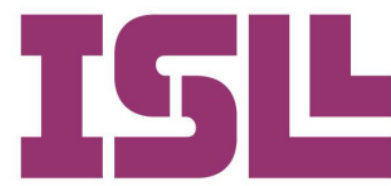

Número 6.

Julio de 2016

\section{La expresión oral y los libros de texto. Estudio de los manuales escolares de Ed. Primaria para conocer el número de actividades orales de las distintas editoriales}

\section{Speaking and textbooks. Study of textbooks of Ed. Elementary for the number of oral activities of different publishers}

\author{
Miriam Suárez Ramírez \\ Universidad de Extremadura
}

Pág. 76 a la 86

\section{Keywords \\ Oral Expression, Text Books, Learning, Teaching Resources}

\begin{abstract}
:
We all know the importance of oral and communication skills and the difficulties presented by our children and youth to make use of them.

On the other hand, we continue checking the importance of exercise textbooks or textbooks in schools. To this it is added that still too often teachers still considers these textbooks or textbooks as the main resource to organize their teaching-learning process.

We believe the textbooks or textbooks remain a very important tool for students and teachers weight. So, what these books or manuals will propose what then put into practice in the school daily.

So we've looked at the primary school textbooks that are present in various colleges Ed. To analyze the extent to propose activities that develop oral expression of students.
\end{abstract}




\section{Introducción}

Las destrezas comunicativas orales de los alumnos de educación primaria están poco trabajadas en el ámbito académico. Esto provoca que los niños y luego jóvenes tengan muchas dificultades para exponer oralmente sus ideas, comentarios, sugerencias, etc.

Aparte de otras causas, una de ellas está relacionada con lo poco que se anima a esta práctica oral desde los libros de texto (el profesor se sigue basando en el libro de texto).

Con esta investigación pretendo analizar los libros de texto implantados en la etapa de primaria en colegios públicos y concertados de la ciudad de Badajoz para evaluar hasta qué punto condicionan el trabajo del profesorado a la hora de realizar actividades que desarrollen la habilidad lingüística oral.

La competencia en comunicación lingüística fijada en el Currículum de Educación Primaria se refiere a la utilización del lenguaje como instrumento tanto de comunicación oral y escrita como de aprendizaje y de regulación de conductas y emociones.

De las cuatro habilidades lingüísticas (hablar, escuchar, leer, escribir) las habilidades orales son las menos trabajadas en la escuela en la etapa de Educación Primaria y las que, luego, mayores repercusiones tienen en adolescentes y jóvenes.

Es habitual escuchar decir que los jóvenes no saben expresarse, exponer en público, argumentar cosas, etc. y, todo ello, como resultado de una formación/enseñanza básica deficitaria o que ha insistido poco en las habilidades orales.

Vivimos en una sociedad en la que se prioriza las habilidades escritas (leer y escribir), que han sido potenciadas por internet y las nuevas tecnologías. Es verdad que los jóvenes usan los chat, los blog, los mensajes de móviles... Todo esto desarrolla las habilidades de leer y escribir, pero hay pocas prácticas en relación con las habilidades orales.

Volviendo a la etapa de primaria, siempre se ha confiado en que, como el niño llegaba a la escuela sabiendo hablar, no era muy necesario insistir en ello, sin embargo, se dedicaba mucho tiempo y esfuerzo a enseñarle a leer y escribir. Todavía es habitual escuchar a los padres decir que llevan a los niños a la escuela para que se les enseñe a leer y escribir.

Igualmente, en muchas ocasiones, con el pretexto de mantener el orden y la disciplina en el aula, los maestros han priorizado el trabajo escolar en silencio, potenciando igualmente la lectura y escritura. 
Por último, parece evidente que el libro de texto o manual escolar sigue siendo el principal recurso utilizado por el maestro. Este recurso condiciona, de manera muy evidente, el trabajo que se desarrolla en el aula. Dependiendo de las actividades o propuestas que se reflejen en los libros de texto, la actividad escolar del aula se dirigirá o se desarrollará mejor en una u otra dirección.

Tomando en consideración todos estos argumentos, he analizado los manuales escolares del área de lenguaje empleados en seis colegios de la ciudad de Badajoz, muestra orientativa de lo que puede ocurrir en los demás centros de la provincia e incluso de la Comunidad Autónoma. Si tenemos en cuenta que esos manuales siguen siendo 'la guía' preferente de los maestros, podemos deducir cómo se organiza el proceso de enseñanza-aprendizaje, qué actividades se priorizan, si se dedica todo el tiempo deseable a las actividades orales, cuáles son las técnicas orales más trabajadas. Estaremos, también, en condiciones de conocer qué editoriales del sector educativo enfatizan más sobre estas actividades orales a la hora de presentar sus propuestas didácticas.

\section{Marco teórico}

Los materiales editados en soporte papel y en especial los llamados libros de texto constituyen uno de los principales recursos para la tarea docente del profesorado, si no el principal recurso en la mayoría de los casos. Podemos afirmar que es el MATERIAL con mayúsculas, el recurso didáctico 'clásico' que ha sobrevivido a reformas y contrarreformas por más que sea cuestionado por administradores y usuarios. Por tanto, son muy relevantes las características didácticas de estos materiales. Por otra parte, el papel real que tienen en el interior de cada aula depende en gran medida del uso específico que de ellos haga el profesor y la profesora correspondiente.

Nadie duda ya que los recursos más utilizados en el siglo XXI se encuentran en formato papel, siendo el libro de texto el material didáctico por excelencia. No obstante, la utilización de los medios por los profesionales de la educación se está viendo incrementada por el uso de recursos relacionados con las TICs, que suelen presentarse normalmente en forma de soportes técnicos o tecnológicos.

La llamada escuela tradicional ha visto siempre el estudio como la fijación en la memoria del contenido de los libros de texto, considerados éstos como los depositarios indefectibles del saber. En una enseñanza de este tipo —denominada comúnmente enseñanza libresca - los niños han de retener multitud de nociones para abocarlas posteriormente en unas pruebas que decidirán su éxito escolar y probablemente, su éxito social.

Cuando el trabajo escolar se reduce a este tipo de aprendizaje, es evidente que al evaluar lo único que se tiene en cuenta es la cantidad de nociones asimiladas por el

ISL, vol. 6, 2016, págs. 76-86 ISSN: 2340-8685
Suárez Ramírez, M. (2016): La expresión oral y los libros de texto. Estudio de los manuales escolares de Ed. Primaria para conocer el número de actividades orales de las distintas editoriales, Investigaciones Sobre Lectura, 6, 76-86. 
alumno. El libro de texto se convierte, entonces, no en un elemento auxiliar de la educación, sino en el instrumento fundamental de la instrucción. Según Cousinet:

Antes, los libros de texto eran sólo un instrumento: el alumno los consultaba y no los leía, como no leemos una guía de ferrocarriles. Se ha querido que lo hiciese y que además obtenga placer haciéndolo. Ya no proporcionan información, sino que intentan educar. Para conseguirlo han multiplicado las ilustraciones, las explicaciones (1980, p. 38).

Podríamos añadir: y ofrecen una guía con una programación válida para todos los grupos con cuestiones y actividades adecuadas a todos los alumnos...

El papel del maestro se ve entonces extraordinariamente facilitado — en sentido negativo - y limitado. De proponer, orientar y corregir las actitudes del alumno, su trabajo pasa a ser de mero intermediario. El libro decide qué, cómo y cuándo se debe enseñar/aprender.

El libro de texto, utilizado como manual único, tiene excesivos inconvenientes y limitaciones. Sin ser exhaustivos, pueden indicarse las siguientes:

- Su tratamiento es unidireccional. Por su estructura intrínseca, no ofrece pareceres diversos ni se plan tea objetivos al margen de la línea establecida.

- Ofrece siempre un saber preestablecido, que acostumbra a nutrirse de estereotipos culturales.

- No desarrolla la formación crítica del niño, ya que no se puede confrontar con otros textos puesto que todos los utilizados son iguales.

- Plantea un ritmo de aprendizaje común. Uniformiza a 30 o 40 niños como sistema para uniformizar a toda la población.

- No se adecúa a nadie en concreto. Es ajeno a cualquier ambiente y a la problemática del grupoclase.

- Pretende la exclusividad del saber. No potencia una pedagogía de la investigación, porque todo está descubierto, todo está experimentado.

- Se presenta como un fin en sí mismo, y no como un medio, un instrumento.

- Su prepotencia puede alejar al alumno de libro y también de la cultura impresa.

- Su contenido queda rápidamente obsoleto; la alternativa no es, evidentemente, un libro más "nuevo".

- Ofrece gran cantidad de información, pero es todavía más la que oculta por motivos económicos (número de páginas, papel, etc.), ideológicos (administración, editorial, autores, etc.), políticos (conveniencias, coyunturas, etc.).

- Abusa de textos informativos expresados con un lenguaje impersonal y abstracto. No suele tener en cuenta la diferencia entre la comprensión de un discurso escrito y uno oral.

- Abusa a menudo el monólogo (salpicado de metáforas y sentimientos del autor).

- Y siempre está mediatizado por un sinfín de intereses: del Editor, de la Administración, del Autor, del "niño-modelo".

Adoptar un solo libro para todos los alumnos y seguirlo sin modificación, rectificación ni ampliación, equivale a esclavizarse a su autor y a privar al niño de múltiples incentivos y posibilidades que desarrollan su pensamiento. En épocas pasadas 
se recomendaba «temer al hombre de un sólo libro» a causa de su visión unilateral y dialéctica radicalmente carente y empobrecedora: pero es todavía peor la escuela de un sólo libro, porque el dogmatismo, el simplismo y la superficialidad son inevitablemente sus consecuencias intelectuales y culturales.

El libro de texto es una construcción que funciona sólo si su contenido motiva, si hace reflexionar; de lo contrario es una obra sin vida. Éste es uno de los más importantes medios de enseñanza para el desarrollo del proceso de enseñanza-aprendizaje, al ser una fuente valiosa de conocimientos y desarrollar las habilidades y hábitos de trabajo independiente.

Si la escuela considera que su finalidad principal es la transmisión de los conocimientos, mediante un maestro que representa el saber oficial a todos los alumnos (que no saben), para que todos alcancen un nivel común de nociones, previamente organizado, el libro de texto es un instrumento necesario y coherente. Pero, si la escuela cree que su principal objetivo es la promoción y el desarrollo cultural (social, afectivo, operativo, también), de cada alumno, hasta los niveles máximos posibles para cada uno; si el único modo de crecer culturalmente consiste en la construcción de conocimientos, hay que reconocer que el libro de texto es un instrumento limitado y limitante, incoherente y que debe ser sustituido. Esta escuela no puede tener como punto de referencia cultural un texto igual para todos sus alumnos, válido para todo el Estado. Se trata más bien de una escuela que acepta el reto de afrontar los pequeños y complejos conocimientos de los alumnos, ligados a su experiencia próxima en el tiempo y en el espacio. Para ello el libro de texto no sirve; sirven los libros, muchos libros: para leer, consultar, contrastar experiencias...

Otro aspecto a destacar son las actividades de expresión oral en los libros de texto. Si partimos de la base de que la función primaria del lenguaje es la comunicación, ésta ha de ocupar un lugar destacado en nuestra programación. A partir del uso del lenguaje que el alumno/a posee, se debe promover y posibilitar distintos empleos del lenguaje en contexto y niveles variados. Se deberán crear situaciones y actividades de aprendizaje en las cuales los alumnos/as sientan la necesidad de emplear formas de lenguaje más elaboradas para ampliar y enriquecer su lenguaje.

En lo que a la expresión oral se refiere, para poder desarrollarla es imprescindible practicarla, es decir, debemos proporcionar al alumno/a oportunidades para expresarse espontáneamente, así como para que transmita a los demás sus ideas, vivencias, sentimientos y opiniones.

Ya sabemos que la función primaria del lenguaje es la comunicación, por eso debe ocupar un lugar destacado en la programación docente. Para ello, son válidas: las

ISL, vol. 6, 2016, págs. 76-86 ISSN: $2340-8685$
Suárez Ramírez, M. (2016): La expresión oral y los libros de texto. Estudio de los manuales escolares de Ed. Primaria para conocer el número de actividades orales de las distintas editoriales, Investigaciones Sobre Lectura, 6, 76-86. 
situaciones de comunicación espontáneas y las planteadas de manera intencionada. Hay que crear situaciones y actividades de aprendizaje en las que los alumnos sientan la necesidad de emplear formas del lenguaje más elaboradas. Nos parece que una forma de organizar las actividades de expresión oral podría ser la que proponen Benita Díez Aguado y M $\mathrm{M}^{\mathrm{a}}$ Isabel Alonso (1993): Expresión de sus vivencias, atención y memoria ante las tareas: (Juegos de eco, juegos de imitación, juegos de acumulación de palabras y juegos de vocabulario), iniciación a la conversación y el diálogo, interpretación de imágenes, narraciones, juegos teatrales espontáneos o dirigidos.

\section{Marco práctico}

\subsection{Objetivos}

1. Conocer y cuantificar las actividades que desarrollan las habilidades lingüísticas orales y escritas, propuestas para ser desarrolladas por diversos manuales escolares (libros de texto).

2. Valorar, por medio de la comparación en relación con otras, el lugar que ocupan las actividades lingüísticas orales en los manuales escolares del área de lengua que se utilizan en las aulas.

3. Comparar los manuales escolares (en lo que se refiere al uso de la expresión oral) utilizados en colegios públicos y concertados.

\subsection{Instrumentos}

Para este estudio hemos utilizado dos cuestionarios:

1. Un cuestionario para la recogida de datos, dirigido al equipo directivo del colegio. Con él se pretende conocer qué manuales escolares son utilizados por ciclos y cursos.

2. Otro cuestionario de análisis de los manuales escolares donde se vayan anotando la categoría de actividades (orales y escritas).

\subsection{Metodología}

En nuestro trabajo hemos tenido en cuenta las siguientes variables:

1. Centros: Público/concertado.

2. Cursos: $1^{\circ}$ a $6^{\circ}$ de Educación Primaria.

3. Ciclos: Los tres de Educación Primaria.

4. Manuales Escolares del área de Lengua Castellana y Literatura por editoriales. 


\subsection{Resultados}

A continuación, mostramos los resultados obtenidos en nuestro trabajo de campo.

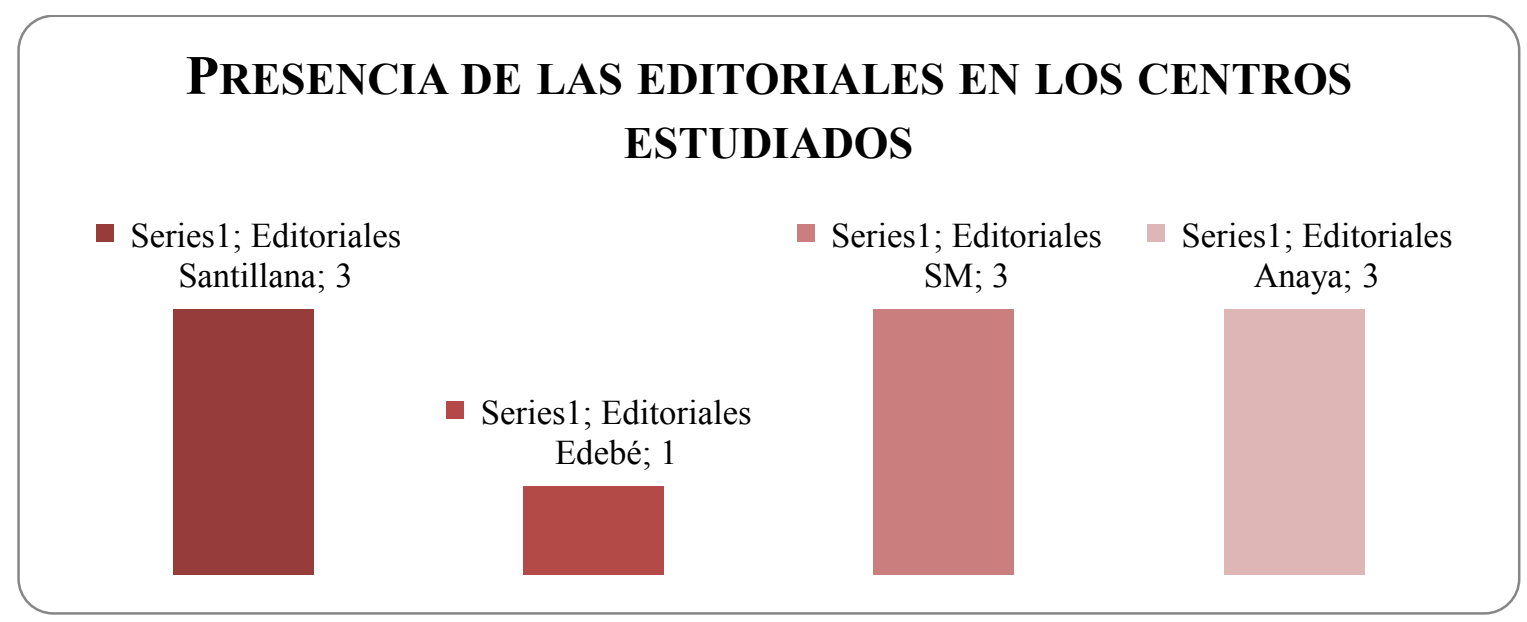

Gráfico 1: Presencia de editoriales en los centros estudiados.

Las editoriales más difundidas por los colegios son Santillana, SM y Anaya, que se dan en tres centros de los seis elegidos, seguidas de Edebé que se da únicamente en un centro.

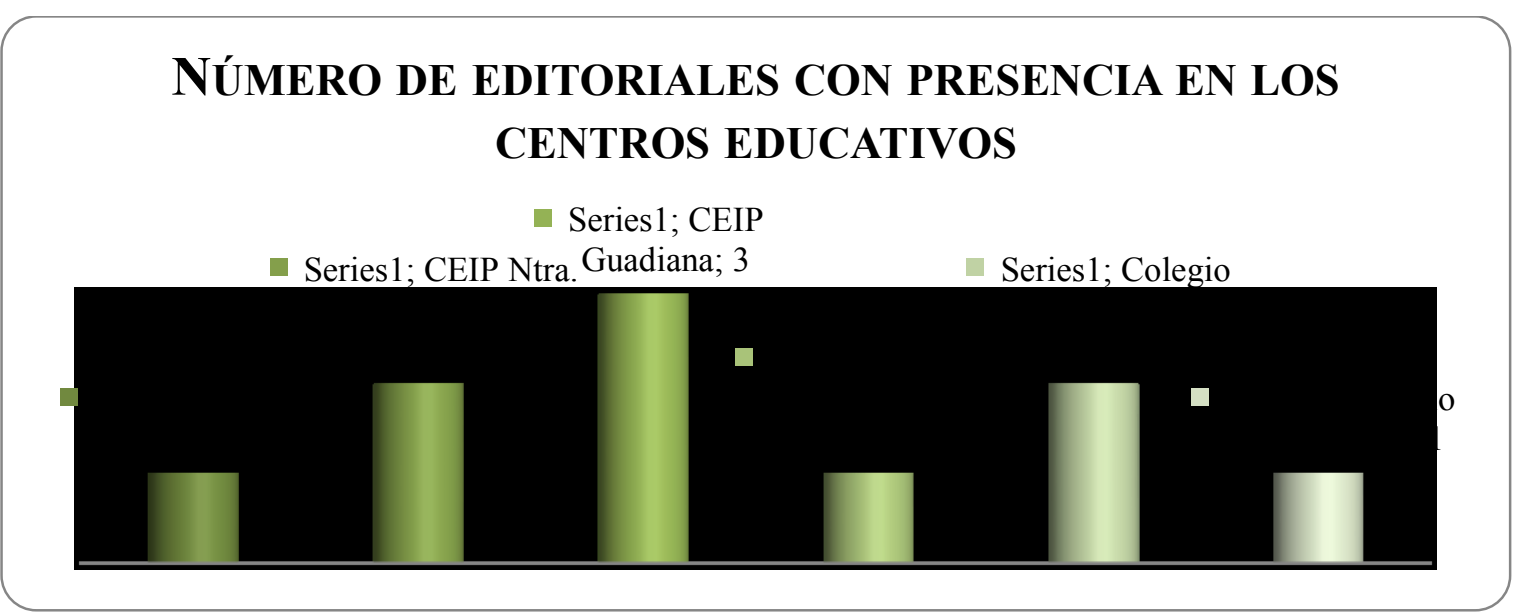

Gráfico 2: Número de editoriales con presencia en los centros educativos

El colegio Guadiana es el centro educativo que recurre a más editoriales a lo largo de la etapa de Ed. Primaria con un total de tres editoriales. El colegio Ntra Sra de la Soledad y el Ntra. Sra. De la Asunción recurren a dos editoriales difertentes, mientras que los colegios Santa Engracia, Salesiano Ramón Izquierdo y Santo Ángel solo apuestan por utilizar una editorial a lo largo de la etapa de Ed. Primaria.

ISL, vol. 6, 2016, págs. 76-86 ISSN: $2340-8685$
Suárez Ramírez, M. (2016): La expresión oral y los libros de texto. Estudio de los manuales escolares de Ed. Primaria para conocer el número de actividades orales de las distintas editoriales, Investigaciones Sobre Lectura, 6, 76-86. 
A continuación, vamos a resumir el número de actividades de cada tipo que, en función de los libros de texto presentes en los diferentes Centros, son sugeridas para ser trabajadas por el profesorado.

\begin{tabular}{|l|c|c|c|c|c|}
\hline EDITORIALES & ORALES & MIXTAS & ESCRITAS & TOTALES & OBSERV. \\
\hline ANAYA & 847 & 795 & 1945 & $\mathbf{3 5 8 7}$ & 6 Libros \\
\hline SANTILLANA & 141 & 117 & 625 & $\mathbf{8 8 3}$ & 2 Libros \\
\hline SM & 1477 & 1470 & 3357 & $\mathbf{6 3 0 4}$ & 8 Libros \\
\hline EDEBÉ & 1048 & 940 & 1803 & $\mathbf{3 7 9 1}$ & 6 Libros \\
\hline TOTALES & $\mathbf{3 5 1 3}$ & $\mathbf{3 3 2 2}$ & $\mathbf{7 7 3 0}$ & $\mathbf{1 4 5 6 5}$ & \\
\hline
\end{tabular}

Tabla1: Actividades-Tipo según editoriales

A la vista de esta tabla observamos que los datos de la Ed. SM y de la editorial Santillana deben ser analizados con cautela porque han sido extraídos de 8 y 2 libros respectivamente, mientras que en el resto de editoriales se han analizado 6, por tanto, requiere por nuestra parte de una ponderación.

Observamos que la editorial que más actividades en total propone es SM incluyendo la ponderación (como si se hubiesen analizado 6 libros) con 6.304 (4.728 cifra ponderada).

Por el contrario. la editorial que menos actividades incluye en sus manuales es Santillana con 883 actividades (puesto que solo se han analizado dos títulos), lo que se mantendría una vez hecha la ponderación correspondiente.

También observamos en esta tabla cómo las actividades escritas se imponen sobre las orales en todas las editoriales, de manera que casi las duplican en el cómputo total (3.513 orales frente a 7.730 escritas). Incluso, en el caso de que añadiéramos a las actividades orales las que nosotros calificamos de actividades mixtas, no superarían el total de actividades escritas (6.835 orales y mixtas frente a 7.730 escritas).

\begin{tabular}{|c|c|c|c|c|c|}
\hline COLEGIOS & ORALES & MIXTAS & ESCRITAS & TOTALES & OBSERV. \\
\hline $\begin{array}{c}\text { SANTA } \\
\text { ENGRACIA }\end{array}$ & 847 & 795 & 1945 & 3587 & $\begin{array}{c}\text { Solo utiliza } \\
\text { Ed. Anaya }\end{array}$ \\
\hline $\begin{array}{c}\text { NTRA.SRA. } \\
\text { DE LA } \\
\text { SOLEDAD }\end{array}$ & 877 & 842 & 2323 & 4042 & Varias Ed. \\
\hline GUADIANA & 1102 & 734 & 2393 & 4229 & Varias Ed. \\
\hline
\end{tabular}




\begin{tabular}{|c|c|c|c|c|c|}
\hline $\begin{array}{c}\text { NTRA. SRA. } \\
\text { DE LA } \\
\text { ASUNCIÓN }\end{array}$ & 1278 & 774 & 2348 & 4400 & Varias Ed. \\
\hline $\begin{array}{c}\text { SANTO } \\
\text { ANGEL }\end{array}$ & 959 & 957 & 2283 & 4199 & $\begin{array}{c}\text { Sólo utiliza } \\
\text { ed.SM }\end{array}$ \\
\hline $\begin{array}{c}\text { SALESIANO } \\
\text { RAMÓN } \\
\text { IZQUIERDO }\end{array}$ & 1048 & 940 & 1828 & 3816 & $\begin{array}{c}\text { Sólo utiliza } \\
\text { ed. Edebé }\end{array}$ \\
\hline TOTALES & $\mathbf{6 1 1 1}$ & $\mathbf{5 0 4 2}$ & $\mathbf{1 3 1 2 0}$ & $\mathbf{2 4 2 7 3}$ & \\
\hline
\end{tabular}

Tabla 2: Actividades totales por tipo y por centros educativos

De la siguiente tabla podemos extraer los siguientes resultados:

En primer lugar, podemos indicar que el centro que utiliza libros de texto que proponen un mayor número de actividades es el colegio Ntra. Sra. De la Asunción $(18,12 \%$ del total) seguido casi con el mismo número por el colegio Guadiana $(17,42 \%$ del total) y por el colegio Santo Ángel (17,29\% del total).

Mientras que en los dos primeros se utilizan varios manuales escolares, en el colegio Santo Ángel solo se utiliza uno lo que refuerza la propuesta de actividades que realiza la editorial SM.

Por el contrario, la editorial Anaya, que es la única editorial analizada en la etapa de primaria en el colegio Santa Engracia, es la que menos actividades incluye en sus manuales $(14,78 \%)$ del total.

Algo parecido ocurre con la editorial Edebé, utilizada en exclusiva para toda la etapa primaria en el colegio Salesiano Ramón Izquierdo con sólo un 15,72\% del total.

En segundo lugar, podemos ver que también el colegio Ntra. Sra. de la Asunción $(20,91 \%)$ es el centro donde los manuales escolares proponen más actividades orales para ser trabajadas a lo largo de la Ed. Primaria (objetivo de esta investigación), seguido del Colegio Guadiana (18\%) y del colegio Salesiano Ramón Izquierdo (17,15\%). El colegio en el que menos actividades orales promueven los libros analizados es el colegio Santa Engracia representando el 13,86\% del total.

En tercer lugar, podemos apreciar cómo el Colegio Guadiana $(18,23 \%)$ es el que, por número de actividades propuestas para ser trabajadas en sus manuales, más fomenta las actividades escritas seguido de Ntra. Sra. De la Asunción (17,90\%) y Ntra. Sra. De la Soledad con el $17,71 \%$ del total.

ISL, vol. 6, 2016, págs. 76-86 ISSN: $2340-8685$
Suárez Ramírez, M. (2016): La expresión oral y los libros de texto. Estudio de los manuales escolares de Ed. Primaria para conocer el número de actividades orales de las distintas editoriales, Investigaciones Sobre Lectura, 6, 76-86. 
En el colegio que menos actividades escritas promueven los libros es Salesiano Ramón Izquierdo con el 13,93\%.

A continuacion, vamos a comparar la propuesta de actividades según la diversa tipologia que venimos considerando, repartida por tipos de centros:

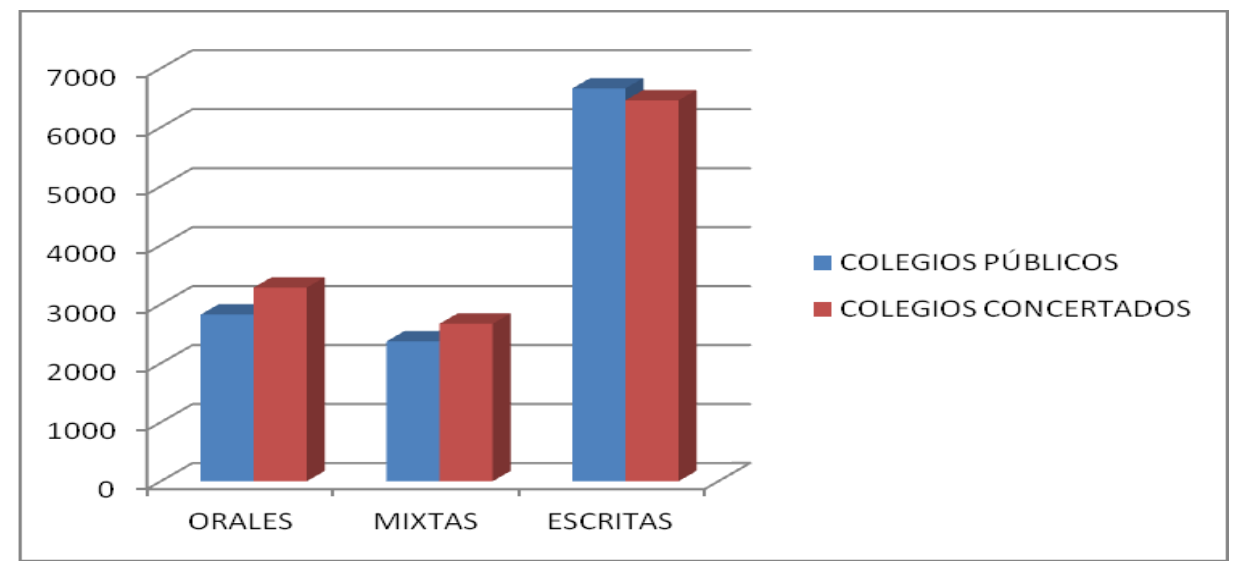

Gráfico 3: Distribución tipología de actividades según Centros

De la siguiente gráfica podemos extraer que las actividades orales y mixtas son más numerosas en los colegios concertados, mientras que las actividades escritas lo son en los colegios públicos.

Podemos apreciar cómo el uso de las actividades escritas se trabaja dos veces más que las actividades orales y mixtas.

\section{Conclusiones}

Tras el análisis de los datos obtenidos estamos en condiciones de elaborar las siguientes conclusiones:

- Las editoriales más implantadas en la ciudad de Badajoz son Santillana, SM y Anaya.

- La editorial que más actividades totales incluye en sus manuales es SM y la que menos Santillana.

- En todas las editoriales predominan las actividades escritas, que duplican a las actividades orales.

- Es poco significativa la diferencia en actividades totales que proponen los libros de texto si se comparan centros públicos con concertados.

- Los colegios concertados utilizan manuales escolares que proponen más actividades orales y mixtas; en cambio los manuales que se utilizan en los colegios públicos proponen un menor número de actividades. 
- El CEIP Guadiana es el que tiene introducidas más editoriales diferentes en la Etapa de Primaria; en cambio el CEIP Santa Engracia y los colegios Santo Ángel y Salesiano Ramón Izquierdo han optado por un solo manual para toda la etapa de Primaria.

- La editorial que menos actividades totales que ofrecen sus manuales es la editorial Anaya, seguida de Edebé.

- Resulta llamativo que en el $1^{\circ}$ ciclo de Ed. Primaria, prolongación de la etapa de Infantil y periodo en que se está iniciando a los alumnos en la lecto-escritura, sea donde los manuales escolares implantados sugieran menos actividades orales.

- De todo el estudio realizado se deduce una cierta despreocupación por parte de los centros educativos a la hora de la elección de los libros de texto o manuales escolares que utilizaran en la etapa de Ed. Primaria.

- También los resultados de nuestro estudio sugieren la necesidad de aumentar el número de actividades orales sobre todo en el $1^{\circ}$ ciclo de Ed. Primaria, como compensación a las propuestas deficitarias que hacen las editoriales.

\section{Bibliografía}

Alvermann, D., Dillón, D. y O’Brien, D. (1990): Discutir para comprender. Ed. Visor: Madrid.

Badía, D y Vilá, M. (1993): Juegos de expresión oral y escrita. Barcelona: Graó.

Ballesteros, C.; Palou, J. (2002): "Les creences dels professors i l'ensenyament de la llengua oral", en Vilá, M. (coord.), Didáctica de la llengua oral formal, Barcelona: Graó, 111-124.

Bally, C. (1932). Lingüistica general y Lingüistica francesa. Madrid: Alianza.

Chomsky, N. (1965): Aspectos de la teoría de la sintaxis, MIT Press, Cambridge, Mass, 4.

Cousinet, R. (1980): Pedagogía del aprendizaje. Barcelona: Planeta.

Díez Aguado, B. y Alonso Íñigo, M. ${ }^{a}$ I. (1993): Lectoescritura. Una propuesta para alumnos con necesidades educativas especiales. Madrid: Escuela Española.

Juárez, A. y Monfort, M. (1989): Estimulación del lenguaje oral. Madrid: Santillana.

Sánchez, A. (1983): Una metodología de la lengua oral. Escuela Española: Madrid.

ISL, vol. 6, 2016, págs. 76-86 ISSN: 2340-8685
Suárez Ramírez, M. (2016): La expresión oral y los libros de texto. Estudio de los manuales escolares de Ed. Primaria para conocer el número de actividades orales de las distintas editoriales, Investigaciones Sobre Lectura, 6, 76-86. 